\title{
A System Architecture Supporting the Agile Coordination of Homecare Services
}

\author{
Elyes Lamine $^{1}$, Sabrina Zefouni ${ }^{1,2}$, Rémi Bastide ${ }^{2}$, and Hervé Pingaud ${ }^{1}$ \\ ${ }^{1}$ Université de Toulouse, EMAC, CGI, Campus Jarlard, 81013 Albi Cedex 09, France \\ \{Elyes. lamine, Herve.pingaud\}@mines-albi.fr \\ ${ }^{2}$ Université de Toulouse, IRIT, CUFR J.F. Champollion, ISIS, Avenue George Pompidou, \\ 81104 Castres, France \\ \{sabrina.zefouni, remi.bastide\}@univ-jfc.fr
}

\begin{abstract}
Dependent people (especially the elderly) are nowadays surrounded by various health services, as well as by social and multi-technology monitoring devices. The PASPORD research project focuses on improving the management of processes involved in home care. These processes are known to be collaborative: their activities spread over different organizations, are carried out by diverse health or logistical actors. These activities must be coordinated by an integrated system, which is the purpose of the present project. However, the deployment of such a process takes place in a very dynamic and uncertain environment. In this paper, we seek to characterize and model this complex process. On the basis of its features, we define the principles of a system architecture to support the collaboration between the stakeholders and provide agility to overcome difficulties induced by its specificity.
\end{abstract}

Keywords: Homecare ecosystem, collaborative process, workflow, information system, eHealth.

\section{Introduction}

Given the increase of 'fragile' persons who require monitoring and care management on a long-term basis, and with the aim of reducing costs of health care, home care is becoming an increasingly common in healthcare delivery. However, in a current trend of increased numbers of elderly persons in need of home cares and shrinking resources within the healthcare sector, these home care providers are facing management challenges in terms of coordination and follow up of activities [1].

It is well-recognized that the establishment of an information system is a major asset to guaranty continuity of care and to enhance its quality. In addition, many reports have underlined that the use of information and communication technologies (ICT) in the performance and management of the health sector is essential for improving the global efficiency of healthcare systems. Therefore, the field of eHealth is currently the subject of intense research projects and commercial development, whose main objective is to design new products or new services to enrich the offer of ICT Health [2-5]. 
However, as concluded in reports on the home healthcare field [1][3-4], today there is not, to our knowledge, a single "killer application" or work addressing the definition of a generic system architecture to support the home care process and allow easy integration of existing ICT health devices or services.

Within this context and in order to provide some answers to the definition of an open and interoperable technology architecture specific to the home care process, the research project PASPORD has been developed. This project, supported by the French region Midi-Pyrénées, aims to define a system architecture supporting the agile coordination of homecare services [8]. It will be experimented across the French region Midi-Pyrénées.

The remaining of this paper is structured in three parts. The first one is focused on a description of the homecare process and its ecosystem. In the second part, the system architecture that we propose to improve is shown. We then present a case study on the use of the proposed system concerning the detection and monitoring of malnutrition in the elderly.

\section{Analysis of Homecare Process and Its Ecosystem}

\subsection{The Organizational Point of View}

Home care includes all health services (medical and para-medical) and social services (cooking, cleaning) provided for the needs of the patient to allow him/her to continue living at home, regardless of age or disability. It is a complex process which represents the chain of various activities achieved by several stakeholder trades (nurse, physician, professional caregivers, etc.) which follow the patient's evolution and needs at home.

Fig.1 shows the ecosystem of the home care process which involves many stakeholders. As shown, multiple exchanges are required to mange and follow up this home care process.

The stakeholders in the homecare process differ widely in terms of their organizational structure, responsibility and technical ability [8]:

- Most of the medical staff involved in homecare in France (doctors, nurses) is selfemployed (liberal profession): they have no hierarchic dependency on the organization that manages the overall homecare process. They usually possess their own information system support, albeit very limited (agenda management, paper or digital patient records, etc).

- Social service (house cleaning and meal delivery) is usually performed by staff that has little computer literacy and are often reluctant to use a computer-based system in their daily work.

- The elderly's relatives often live far away, paying him/her irregular visits. However, they wish to keep in touch with their parent via telephone and be kept informed on his/her health state.

- Finally, the homecare system is funded by regional public units, which are billed for the work of the various stakeholders. These units need to be reliably informed of the actual work that has been performed, time spent at home by the various stakeholders, etc. 


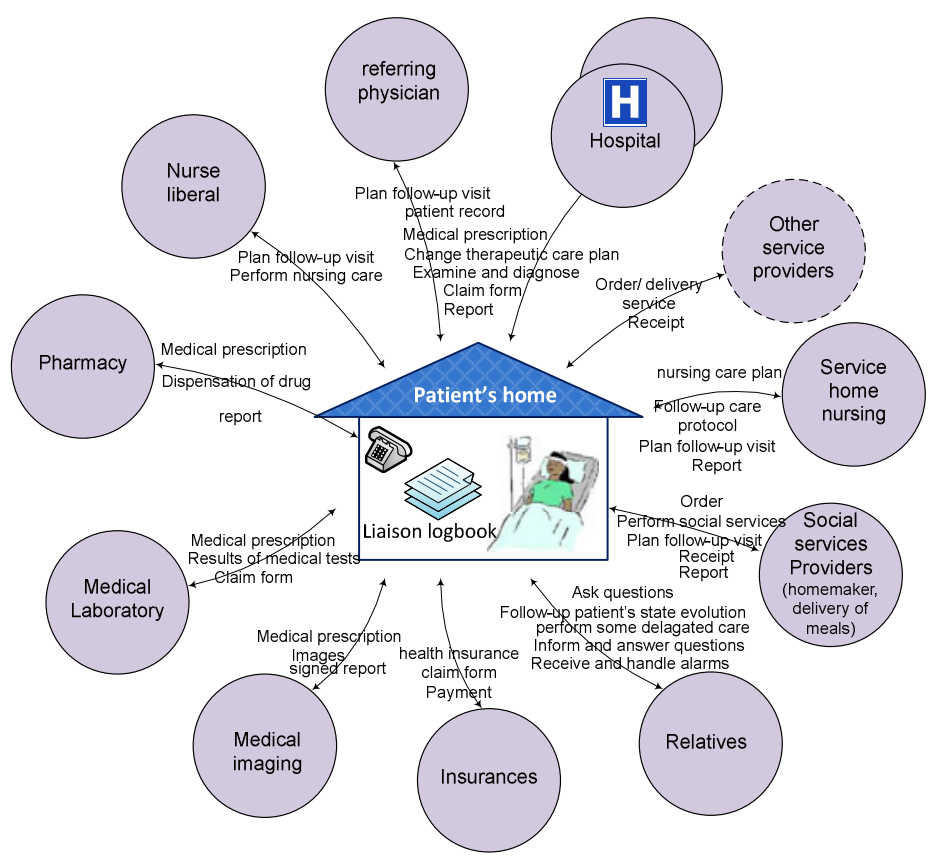

Fig. 1. Home care ecosystem

In France, homecare is mainly managed by regional associations that coordinate the activity of all the stakeholders involved (doctors, nurses, meal delivery, etc.).

\subsection{Informational Point of View}

The coordination of these numerous stakeholders with heterogeneous profiles and trades is definitely required to assure the quality and the continuity of care. Two levels of coordination in the homecare are notable:

- Internal coordination between stakeholders from the same organization or the same service. They use a shared Information System (IS) and act towards the same goal.

- External coordination requires interacting and coordinating actors and heterogeneous IS belonging to the various organizations involved in the homecare. Several upcoming services such as future electronic medical record will be required to interact with the systems already in place.

Currently, the activity of all these persons is mainly managed manually, using a socalled 'liaison logbook'. It is generally an unstructured, physical notebook that is kept at the elder's home and used for communication between the family and the various stakeholders, as well as between stakeholders. Each stakeholder notes the date of the visit, the operations performed, recommendations or any other form of comment. Fig.1 illustrates the central role played by the liaison logbook in the various processes 
of care at home. The logbook is the primary means of communication between different stakeholders.

It is noteworthy that the liaison logbook contains very useful data, underused in the homecare process. Indeed, it stores information of different types: logistical information which helps for the coordination of activities, medical information, general information on the patient, any information which may help improve the quality of the homecare and possibly the detection of the patient's health change via an intelligent monitoring system [8].

\section{Proposed Architecture System}

\subsection{Screening Relevant Elements to Be Considered}

Currently, the home care ecosystem is still in a primary stage of maturity with a lack of adequate access to information and of communication between stakeholders which hampers the management and the follow up of the home care process.

This gap results in a weak use of the ICT tools and services and an insufficient interoperability between these services and the information system of the different stakeholders. The idea is thus not to develop new ICT products, but to think how to make them useful and integrate them in a consistent common scenario covering a wide range of home care situations. This is the main purpose of the research project PASPORD.

In order to target and facilitate the identification of components of our proposed architecture, two major findings are pointed out:

- Even though the patient in home care receives daily visits from the different stakeholders, it sometimes occurs that he/she spends a long time without being visited by any of them. This could be problematic especially in the case of elderly patients who are subject to falls, discomfort and many serious domestic incidents. It is obvious that improving homecare implies a better detection and prevention of incidents or of the degradation of the elder's health.

- In order to assure the quality of care, relevant information such as alerts, personal to-do list, incident reports, organization requests, update of the patient's plan care or records, etc., should be accessible to the most appropriate actor of the home care circuit (doctor, nurse, emergency service, neighborhood, relatives, etc.) at the right time. This implies improved means of communication and coordination of stakeholders to overcome the limitations of the current liaison logbook.

\subsection{Target Architecture}

In order to achieve the two objectives mentioned above, we propose as shown in Fig. 2, an architecture that includes a surveillance system in order to detect dangerous situations, combined with an agile system of automatic exchange of information to coordinate the different performances. The whole is supported by multi-modal communication systems, such as a combination between personal digital assistants (PDA) and tablet PCs). 


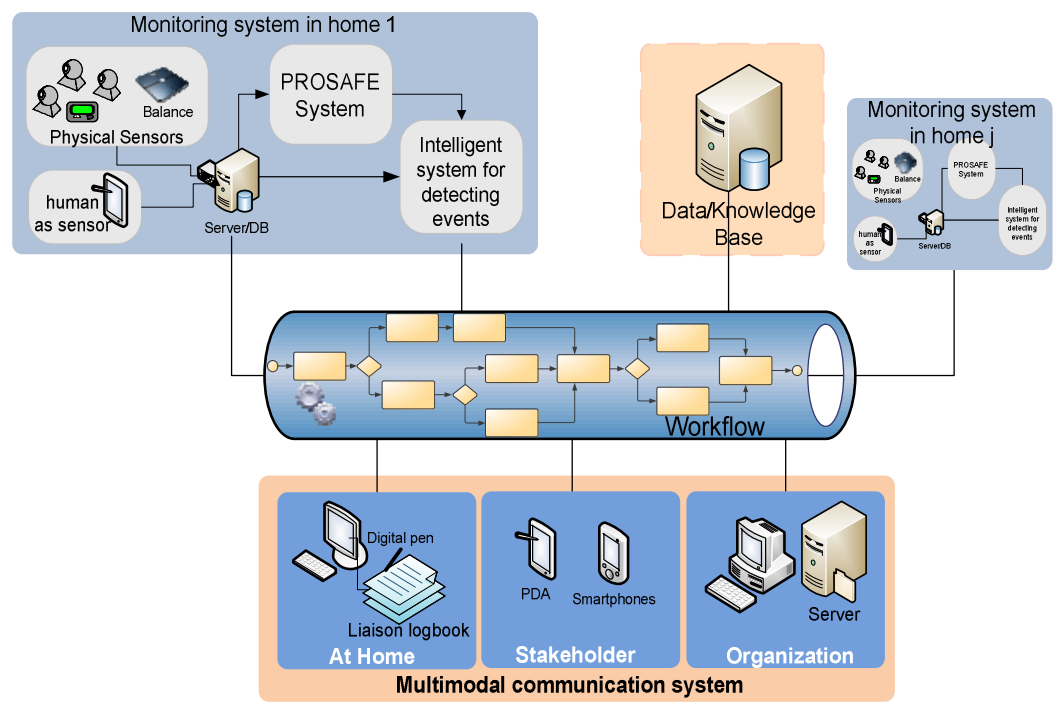

Fig. 2. Overall Architecture of the PASPORD project

The architecture of choice that we propose here, is composed of:

1. PROSAFE which consists of a monitoring system for dependent people. It was designed in the laboratory LAAS in Toulouse, one of the project partners. This system has been tested on several sites such as a retirement home and an apartment. This system can warn of certain dangers in real time (fall, fugue, etc.), based on the learning habits of the person under surveillance [10].

2. An intelligent system for detection of relevant events using Multi-Agent System (MAS), an organized set of agents [11]. An agent is a software entity or person, which are assigned a certain task that they can perform independently and in cooperation with other agents. The purpose of this multi-agent system is to prevent, on the long-term, degradation of the patient's status. This is based on a classification made by agents regarding the parameters monitored.

3. The database saves patient information as it does at present, in addition to actimetry information provided by the monitoring equipment installed at home (e.g. stirring overnight, movement speed, etc.), and transmissions made between different stakeholders, in order to track them easily at any time.

4. The workflow engine [9][12] based on the WfMC (Workflow Management Coalition Specification) is the automation process in which documents, information and tasks move from one part to another according to a set of rules. In other words, it consists of a system that allows the coordination of work among several participants following a predefined process model. Its role is to coordinate the work involved by transmitting the necessary information and tasks to different stakeholders, depending on the condition of the patient's treatment plan and the role of the interveners. 


\section{Case Study}

We propose a case study on the detection and monitoring of malnutrition for the elderly. According to a study conducted by the French HAS (Haute Autorité de Santé: High Health Authority) in 2007, cases of malnutrition were observed in 350000 to 500000 elderly people living at home and at least between 100000 and 200000 living in institutions [15]. These numbers will tend to grow as the population continues to age. Malnutrition among the elderly has dramatic consequences -the risk of death is two to four times higher. Malnutrition exacerbates the health problems of the elderly person and increases the risk of falls, dehydration, mental disorders, and time required for healing. In addition, it generates enormous healthcare costs at the expense of the whole society. According to the causes of undernutrition, the treatment plan is different. This means that according to the patient's status the process should be adapted differently.

The best way to combat malnutrition is to detect it as early as possible. This can be done by early detection before it has negative consequences on the health of the elderly or even by avoiding people at risk. There are simple ways to detect malnutrition. Most studies and general practitioners [13-16] agree on screening for malnutrition based on some criteria such as: the change in weight over time, body mass index, the MNA test result [16], etc.

In the table 1, largely inspired by that of the HAS[13], We present the different thresholds for alerting undernutrition, distinguishing two types of malnutrition: undernutrition and the severe malnutrition that requiring medical intervention.

Table 1. Characterization of under nutrition and malnutrition

\begin{tabular}{l|l}
\hline Under nutrition & Severe malnutrition \\
\hline $\begin{array}{l}\text { Weight loss of 5\% in a month } \\
\text { or } 10 \% \text { in } 6 \text { months }\end{array}$ & $\begin{array}{l}\text { Weight Loss: } \geq 10 \% \text { in a month } \\
\text { or } \geq 15 \% \text { in } 6 \text { months }\end{array}$ \\
\hline Body Mass Index $<21$ & BMI $<18$ \\
\hline Albumin $<35 \mathrm{~g} / 1$ & Albumin $<30 \mathrm{~g} / 1$ \\
\hline Cholesterol $<1.6 \mathrm{~g} / 1$ & \\
\hline $17<$ MNA Global $<23,5$ & MNA Global $<17$ \\
\hline
\end{tabular}

The system proposed in the PASPORD project helps detecting malnutrition as soon as possible:

- Preventing measures can be taken by informing family members of a potential risk of malnutrition and by displaying the evolution of weight and body mass index to nurses visiting the patient.

- In other cases, through automatic monitoring of weight, an alert will be triggered whenever there would be an indication of malnutrition. This warning will trigger the appropriate process to the origins of the problem and how to treat it. The process of screening and treatment of malnutrition in the elderly is shown in Fig.3.

We chose to model this process with BPMN (Business Process Modeling Notation). Five pools represent five different categories of actors involved in the process: the monitoring system that triggers the alarm as the system environment. 
The nurses' aide, coordinator and family as business actors, and a central line (workflow) which represents the sequence of activities in the process of screening and treatment of malnutrition.

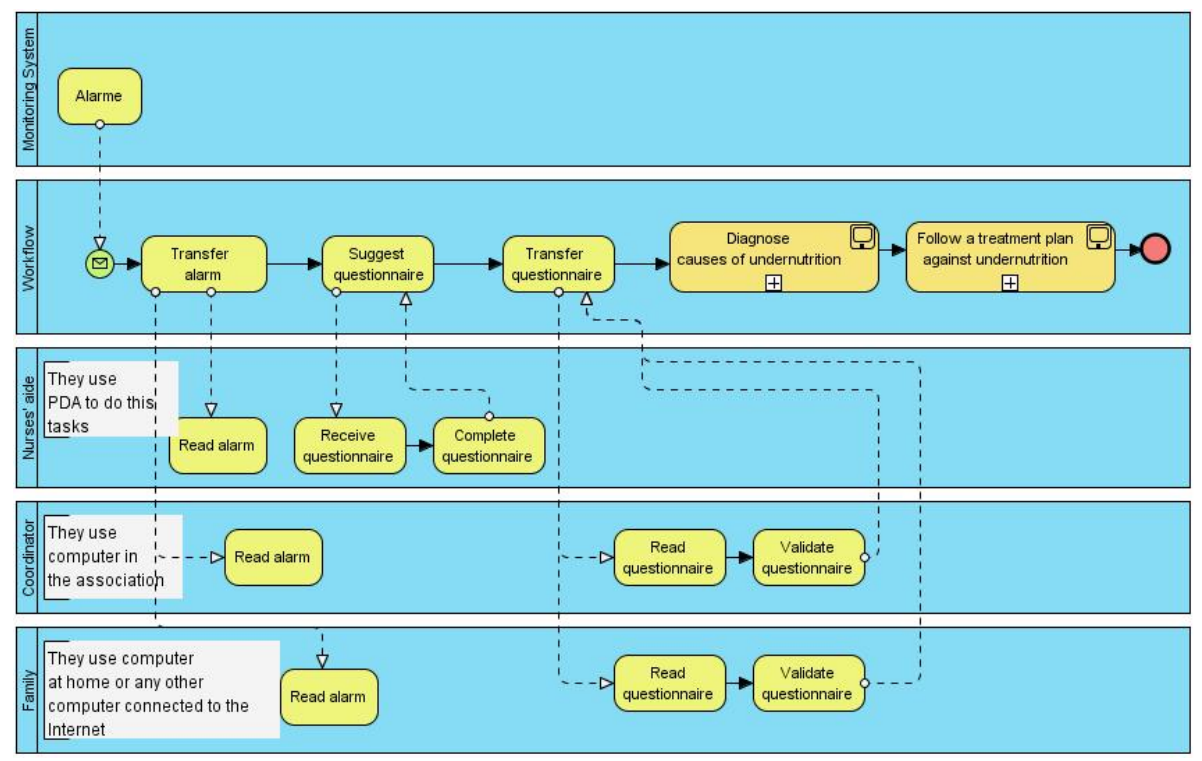

Fig. 3. Overall process management of malnutrition

In summary, the process of screening and treatment of malnutrition follow these steps:

1. Detecting malnutrition: when the person is weighed, compare his current weight with his past weight record of the previous period. Trigger an alarm if malnutrition is suspected.

2. Preventing medical staff and family: alert stakeholders of the malnutrition problem.

3. Diagnose the cause of weight loss: diagnosis begins with a questionnaire that helps fill nursing and will be forwarded to the nurse coordinator. The family then decides to inform the treating physician.

4. After the intervention of a doctor and discussion between the family and the nurse coordinator, a new treatment plan will be implemented to allow the resumption of weight lost.

\section{Conclusion}

In this paper we present an overall architecture of a collaborative system to improve homecare. This architecture is a coupling system of monitoring and coordination system which helps to ensure the safety of the elderly and facilitates coordination among stakeholders. The innovative idea is the use of human actors as sensors that 
transmit information on the status of the elderly. This information feeds the progress of the workflow and helps prevent some deterioration in the elderly's condition. The next step in our work is the implementation of a flexible and adaptive inter-organizational workflow in which we will define connections between the business process of homecare and this workflow.

\section{Acknowledgment}

The work presented here, as well as the PhD. of Sabrina Zefouni, is mainly funded by French Region Midi-Pyrénées, with support from University J.F. Champollion and Castres Syndicate for Higher Education and Research.

\section{References}

1. FNEHAD: Livre blanc des systèmes d'information en hospitalisation à domicile, white paper (Juin 2009)

2. Boulos, M.K., Lou, R.C., Anastasiou, A., Nugent, C.D., Alexandersson, J., Zimmermann, G., Cortes, U., Casas, R.: Connectivity for Healthcare and Well-Being Management: Examples from Six European Projects. Int. J. Environ. Res. Public Health 6(7), 1947-1971 (2009)

3. Lasbordes, P.: La télésanté: un nouvel atout au service de notre bien-être

4. Gartner: eHealth for a Healthier Europe (Juin 2009)

5. Alexander, G.L., Wakefield, D.S.: Information Technology Sophistication in Nursing Homes. Journal of American Medical Directors Association 10(6), 398-407 (2009)

6. Guinet, A., Ladet, P., Marcon, E.: 2HM: Hospitalisation Hors les Murs. Journées STP d'Aix en Provence, CNRS (2007)

7. Liu, C., Li, Q., Zhao, X.: Challenges and opportunities in collaborative business process management: Overview of recent advances and introduction to the special issue. Springer Science and Business Media, LLC, Heidelberg (2008)

8. Bastide R., Zefouni S., Lamine E.: The Homecare Digital Ecosystem: an Information System Support Architecture. In: IEEE DEST 2010, Dubai, EAU (2010)

9. Van der Aalst, W.M.P., Jablonski, S.: Dealing with Workflow Change: Identification of issues and solutions. International Journal of Computer Systems, Science, and Engineering (2000)

10. Bonhomme, S., Campo, E., Esteve, D., Guennec, J.: PROSAFE-extended, a telemedicine platform to contribute to medical diagnosis. Journal of Telemedicine and Telecare 14(3) (May 2007)

11. Rammal, A., Trouilhet, S., Singer, N., Pecatte, J-M. : An Adaptive System for Home Monitoring Using a Multiagent Classification of Patterns. International Journal of Telemedicine and Applications (2008)

12. Hollingsworth, D.: TheWorkflow Reference Model, Technical report wfmctc- 1003, Workflow Management Coalition (1995)

13. HAS: Stratégie de prise en charge en cas de dénutrition protéino-énergétique chez la personne âgée (2007), http://www.has-sante.fr/portail/jcms/C_546549/ malnutrition-in-the-elderly-nutritional-support-strategy

14. Pirlich, M., Lochs, H.: Nutrition in the elderly. Best Practice \& Research Clinical Gastroenterology, 868-884 (2001)

15. David, B., Reuben, M.D.: Quality Indicators for the Care of Undernutrition in Vulnerable Elders. Jags 55(S2) (October 2007)

16. Guigoz, Y., Vellas, B.: The Mini Nutrition Assessment (MNA) for grading the nutritional state of elderly patients: presentation of the MNA, history and validation. In: Nestlé nutrition workshop series clinical \& performance, vol. 1, pp. 3-12 (1999) 